\title{
Dynamical Analysis of Predator-Prey Model Leslie-Gower with Omnivore
}

\author{
Rina Exviani ${ }^{1 *}$, Wuryansari Muharini Kusumawinahyu², Noor Hidayat ${ }^{3}$ \\ ${ }^{1}$ Master Program of Mathematics, Faculty of Mathematics and Natural Sciences, University of Brawijaya, Malang, \\ Indonesia \\ ${ }^{2}$ Department of Mathematics, Faculty of Mathematics and Natural Sciences, University of Brawijaya, Malang, Indonesia
}

\section{Abstract}

This article discussed a dynamical analysis on a model of predator-prey Leslie-Gower with omnivores which is modified by Lotka-Volterra model with omnivore. The dynamical analysis was done by determining the equilibrium point with its existing condition and analyzing the local stability of the equilibrium point. Based on the analysis, there are seven points of equilibrium. Three of them always exist while the four others exist under certain conditions. Four points of equilibrium, which are unstable, while the others three equilibrium point are local asymptotically stable under certain conditions. Moreover, numerical simulations were also conducted to illustrate the analytics. The results of numerical simulations agree with the results of the dynamical analysis.

Keywords: local stability, omnivore, predator-prey models, the equilibrium point.

\section{INTRODUCTION}

Lotka-Volterra model was firstly introduced Lotka in 1925 and Volterra in 1926 [1]. LotkaVolterra's study has produced a simple model of predation or interaction between two species in an ecosystem. They also have introduced classical Lotka-Volterra model, which is currently developed by researchers [2].

In 1948, Leslie discussed Lotka-Volterra model and found impossibility in a model, which is infinity in predator growth [3]. Therefore, Leslie and Gower introduced a new name's predatorprey model, which is modification of LotkaVolterra's model. The model is known as LeslieGower Predator-Prey Model. Leslie-Gower have modified Lotka-Volterra's predator-prey model by assuming that the predation of predator is limited, which means that the predation of predator will not more carrying capacity of prey. Leslie-Gower two dimension models as:

$$
\begin{aligned}
& \frac{d x}{d t}=\left(x_{1}-a_{1} y-b_{1} x\right) x_{x} \\
& \frac{d y}{d t}=\left(x_{2}-a_{2} \frac{y}{x}\right) y .
\end{aligned}
$$

with $x(t)$ state the population density of prey and $y(t)$ state the population density of predator.

In 2015, Andayani and Kusumawinahyu [4] a three species predator - prey model, the third

\footnotetext{
* Correspondence address:

Rina Exviani

Email : rhinaexviani@gmail.com

Address : Dept. Mathematics, Faculty of Mathematics and Natural Science, University of Brawijaya, Veteran Malang, Malang 65145.
}

species are omnivores. This model is constructed by assumming there are just three species in such an ecosystem. The first species, called as prey (rice plant), the prey for the second and the third species. The second species, called as predator (carrion), only feeds on the first species and can extinct with prey. The third species, namely omnivores (mouse), eat the prey and carcasses of predator. Consequently, omnivores of predator only reduces the prey population but does not affect the predator growth. Assumed that the prey population grow logistically and any competition between omnivores [5]. Based on these assumption, the mathematical model representing those growth density of population rates by nonlinear ordinary differential equation system, namely

$$
\begin{aligned}
& \frac{d x}{d t}=x(1-y-z-b x) . \\
& \frac{d y}{d t}=y(-c+x) \\
& \frac{d z}{d t}=z(-e+f x+g y-\beta z) .
\end{aligned}
$$

In this model, $x(t), y(t)$ and $z(t)$ the density of prey, predator, and omnivore populations, respectively. All parameter of model (2) are positive. The death rates of the predator and omnivore are denoted by $c$ and $e$, respectively. The parameter $f$ rivalry toward prey that effect increases of omnivore population, while the parameter $g$ rivalry toward prey that effect increases of omnivore population. Parameter $b$ and $\beta$ carrying the capacity of the prey and omnivore, respectively [5]. The aim of this study is a dynamical analysis on a model of predator-prey 
Leslie-Gower with omnivores which is modified by Lotka-Volterra model with omnivore.

\section{MATERIAL AND METHOD}

In this study, predator-prey model by LeslieGower with omnivore. This model is constructed by assumming the third species are omnivores. This model is constructed by assumming there are just three species in such an ecosystem. The first species, called as prey (rice plant), the prey for the second and the third species. The second species, called as predator (carrion), only feeds on the first species and can extinct with prey. The third species, namely omnivores (mouse), eat the prey and carcasses of predator. Consequently, omnivores of predator only reduces the prey population but does not affect the predator growth. Assumed that the prey population grow logistically and any competition between omnivores.

\section{Literature Study}

Literature study related to the research process, such as the literature discussing the LeslieGower model, Lotka-Volterra model, omnivore, and forward-backward sweep method [7-12]. We also used other supporting references in problem solving in this study. In the Lotka-Volterra predator-prey model with omnivore, namely:

$$
\begin{aligned}
& \frac{d x}{d t}=x(1-y-z-b x) x \\
& \frac{d y}{d t}=y(-c+x) x \\
& \frac{d z}{d t}=z(-e+f x+g y-\beta z) .
\end{aligned}
$$

on this system has only five equilibrium point's, namely:

$$
\begin{aligned}
& E_{0}=(0,0,0) \\
& E_{1}=\left(\frac{1}{b}, 0,0\right) \\
& E_{2}=(c, 1-b c, 0) \\
& E_{3}=\left(\frac{e+\beta}{f+\beta b}, 0, \frac{f-b e}{f-\beta b}\right) \\
& E_{4}=\left(c \frac{\beta(1-b c)-(f c-e)}{g+\beta}, \frac{g(1-b c)+f(f c-e)}{g+\beta}\right)
\end{aligned}
$$

To accommodate biological meaning, the existence conditions for the equilibria require that they are nonnegative. It is obvious that $E_{0}$ dan $E_{1}$ always exist, $E_{2}$ exist if $b c<1, E_{3}$ exist if $f>b e$ and $E_{4}$ the densities of omnivores and predators $1-b c$ has to be positive. Then, $E_{4}$ exist if $b c<1$ and $0<f c-e<\beta(1-b c)$.

While, predator-prey model by Leslie-Gower with omnivore has seven equilibrium points (Table 1). So the predator-prey model by LeslieGower model with omnivores is more concrete in this case.

Table 1. Equilibrium Points of predator-prey model by Leslie-Gower with omnivore

\begin{tabular}{ll}
\hline \multicolumn{1}{c}{$\begin{array}{c}\text { Equilibrium } \\
\text { Points }\end{array}$} & \multicolumn{1}{c}{ Existence Requirement } \\
\hline$E_{1}=(0,0,0)$ & - \\
$E_{2}=\left(0, \frac{k r_{2}}{a_{2}}, 0\right)$ & - \\
$E_{3}=\left(\frac{r_{1}}{b_{1}}, 0,0\right)$ & - \\
$E_{4}=\left(0, y_{4}, z_{4}\right)$ & $c_{3} k r_{2}>a_{2} c_{1}$ \\
$E_{5}=\left(x_{5}, 0, z_{5}\right)$ & $r_{1} c_{2}>b_{1} c_{1}$ \\
$E_{6}=\left(x_{6}, y_{6}, 0\right)$ & $a_{2} r_{1}>b_{2} k r_{2}$ \\
$E_{7}=\left(x_{7}, y_{7}, z_{7}\right)$ & $c_{4} r_{1}+c_{1} b_{3}>\frac{c_{1}}{c_{3}}\left(b_{2} c_{4}+b_{3} c_{3}\right)$ \\
\hline
\end{tabular}

\section{MATHEMATICAL MODEL}

This study constructs Lotka-Volterra's predator-prey model with omnivore (2). This model is developed by modify the predator that previously used Lotka-Volterra's form to Leslie-Gower's, which was examined by Leslie-Gower. This is based on the fact that predator depends on the available number of prey to establish. Therefore, the model is stated to be in the following equation system (3):

$$
\begin{aligned}
& \frac{d x}{d t}=x\left(r_{1}-b_{1} x-b_{2} y-b_{2} z\right), \\
& \frac{d y}{d t}=y\left(r_{2}-\frac{a_{2}}{x+k} y\right) . \\
& \frac{d z}{d t}=z\left(-c_{1}+c_{2} x+c_{3} y-c_{4} z\right)
\end{aligned}
$$

with $x=x(t), y=y(t)$, and $z=z(t)$ state the population density of prey, predator, and omnivore. All of the parameters are positive in value. Parameters $r_{1}$ and $r_{2}$ respectively show intrinsic growth of prey and predator. $b_{1}$ is the coeffcient of competition between prey, $b_{2}$ is the predator interaction coefficient between predator to prey and $b_{3}$ is a predatory interaction between omnivores against prey. Whereas $a_{2}$ is an interaction parameter between predators and parameter $k$ is a parameter of protection against 


\section{(Exviani et al)}

predators. Parameter $c_{1}$ is omnivorous natural death, $c_{2}$ partially omnivorous predictor coefficient to prey, $c_{3}$ as predator coefficient of predator carcass, $c_{4}$ is competition between omnivorous population. While, tribal form $\frac{\left(a_{2} y\right)}{(x+k)}$ can be interpreted as scarcity of prey may stimulate predators to replace foot sources with other alternatives. Therefore, it is assumed that predators depend not only on prey, but predators can eat other than prey in the prey environment. So in this article it is modeled by adding a positive constant $k$ to the division.

\section{RESULT AND DISCUSSION}

All parameters of Equation (3.1) in this study are assumed positive in value. Parameters $r_{1}$ and $r_{2}$ consecutively show intrinsic growth of prey and predator. $b_{1}$ is the competition coefficient among preys, $b_{2}$ is the predation interaction coefficient between predator and prey, and $b_{a}$ is the predation interaction between omnivore and prey. Meanwhile, $a_{2}$ is the interaction parameter among predators, and parameter $k$ is the protection parameter against predator. Parameter $c_{1}$ is the natural death of omnivore, $c_{2}$ is the predation coefficient of omnivore on prey, $c_{3}$ is the predation coefficient on the carcass of predator, and $c_{4}$ is the competition among omnivore populations.

\section{Equilibrium Point and Existence}

The point of Equilibrium (3) is solution for sytem:

$$
\begin{array}{r}
x\left(r_{1}-b_{1} x-b_{2} y-b_{3} z\right)=0 \\
y\left(r_{2}-\frac{a_{2}}{x+k} y\right)=0 \\
z\left(-c_{1}+c_{2} x+c_{2} y-c_{4} z\right)=0 .
\end{array}
$$

The system has seven points of equilibrium, namely $E_{1}=(0,0,0), \quad E_{2}=\left(0, \frac{k r_{2}}{a_{2}}, 0\right)$, and $E_{a}=\left(\frac{r_{1}}{b_{1}}, 0,0\right)$ in which the three points exist unconditionally, equilibrium point $E_{4}=\left(0, \frac{r_{2} k}{\sigma_{2}}, \frac{c_{3} r_{2} k-c_{1} \sigma_{2}}{c_{4} a_{2}}\right)$ exists with the condition of $c_{2} r_{2} k>a_{2} c_{1}$, equilibrium point

$$
\begin{aligned}
& E_{5}=\left(\frac{r_{1} c_{4}+b_{3} c_{1}}{c_{2} b_{32}+c_{4} b_{1}}, 0, \frac{c_{2} y_{1}-c_{1} b_{1}}{c_{2} b_{32}+c_{4} b_{1}}\right) \text { exists if } \\
& z=c_{2} r_{1}>c_{1} b_{1}, \quad \text { equilibrium point } \\
& E_{6}=\left(\frac{r_{1} a_{2}-b_{2} r_{2} k}{a_{2} b_{1}+b_{2} r_{2}}, \frac{r_{2}}{a_{2} b_{1}+b_{2} r_{2}}, 0\right) \text { exists if } \\
& a_{2} r_{1}>b_{2} k r_{2} \text {. }
\end{aligned}
$$$$
\text { point } E_{7}=\left(x_{70} y_{7}, Z_{7}\right) \text { with }
$$

$$
\begin{aligned}
& x_{7}=\frac{a_{2} r_{1} c_{4}+a_{2} c_{1} b_{3}-r_{2} k b_{2} c_{4}-r_{2} k c_{3} b_{3}}{a_{2} b_{1} c_{4}+a_{2} c_{2} b_{3}+r_{2} b_{2} c_{4}+r_{2} c_{3} b_{3}} \\
& y_{7}=\frac{r_{2}\left(r_{1} c_{4}+c_{1} b_{3}+k b_{1} c_{4}+k c_{2} b_{3}\right)}{a_{2} b_{1} c_{4}+a_{2} c_{2} b_{3}+r_{2} b_{2} c_{4}+r_{2} c_{3} b_{3}} \\
& z_{7}=\frac{r_{1} r_{2} c_{3}+r_{1} a_{2} c_{2}+b_{1} r_{2} k c_{3}-b_{1} a_{2} c_{1}-b_{2} r_{2} c_{1}-b_{2} r_{2} k c_{2}}{r_{2} b_{2} c_{4}+r_{2} c_{3} b_{3}+a_{2} b_{1} c_{4}+a_{2} c_{2} b_{3}} \\
& \text { exists if } r_{1} c_{4}+b_{3} c_{1}>\frac{c_{1}}{c_{3}}\left(b_{2} c_{4}+b_{3} c_{3}\right) .
\end{aligned}
$$

\section{Stability Analysis}

The local stability of system (3) for each equilibrium point is as follows.

a) $E_{1}=(0,0,0)$ is unstable

b) $E_{2}=\left(0, \frac{r_{2} k}{a_{2}}, 0\right)$ is stable if $r_{1} a_{2}<b_{2} r_{2} k$ and $c_{2} r_{2} k>c_{1} a_{2}$

c) $E_{3}=\left(\frac{r_{1}}{b_{1}}, 0,0\right)$ is unstable

d) $E_{4}=\left(0, \frac{r_{2} k}{a_{2}}, \frac{c_{3} r_{2} k-c_{1} a_{2}}{c_{4} a_{2}}\right)$ is stable if $c_{4} x_{1}+c_{1} b_{3}<\frac{k x_{2}}{a_{2}}\left(b_{2} c_{4}+b_{3} c_{3}\right)$

e) $E_{5}=\left(\frac{r_{1} c_{4}+b_{3} c_{1}}{c_{2} b_{3}+c_{4} b_{1}}, 0, \frac{c_{2} x_{1}-c_{1} b_{1}}{c_{2} b_{3}+c_{4} b_{1}}\right)$ is unstable

f) $E_{6}=\left(\frac{r_{1} a_{2}-b_{2} r_{2} k}{a_{2} b_{1}+b_{2} r_{2}}, \frac{r_{2}\left(r_{1}+b_{1} k\right)}{a_{2} b_{1}+b_{2} r_{2}}, 0\right)$ is unstable

g) $E_{7}=\left(x_{7 x} y_{7}, z_{7}\right)$.

This stability analysis uses the criteria of Routh-Hurwitz $\lambda^{3}+A \lambda^{2}+B \lambda+C=0$. The characteristic equation will have negative roots if and only if $A>0, C>0$ and $A B>C$. Therefore, it can be concluded that $A B-C>0$. If the condition is met, the equilibrium point of $E_{7}$ will be stable.

\section{Proof}

i. Jacobi matrix system (3) for $E_{1}$ is $f\left(E_{1}\right)=\left[\begin{array}{ccc}r_{1} & 0 & 0 \\ 0 & r_{2} & 0 \\ 0 & 0 & -c_{1}\end{array}\right]$. The three Eigen values of the matrix $f\left(E_{1}\right)$ are positive, so $E_{1}$ is unstable.

ii. Jacobi matrix $E_{2}=\left(0, \frac{r_{2} k}{a_{2}}, 0\right)$ is

$$
\begin{aligned}
& l=\left[\begin{array}{ccc}
r_{1}-\frac{b_{2} r_{2} k}{a_{2}} & 0 & 0 \\
\frac{r_{2}^{2}}{a_{2}} & -r_{2} & 0 \\
0 & 0 & -c_{1}+\frac{c_{2} r_{2} k}{a_{2}}
\end{array}\right] \text {, } \\
& \text { which has eigen values } \\
& \lambda_{1}=r_{1}-\frac{b_{2} r_{2} k}{a_{2}}, \lambda_{2}=-r_{2}, \lambda_{\mathrm{a}}= \\
& -c_{1}+\frac{c_{3} r_{2} k}{a_{2}}
\end{aligned}
$$


- The equilibrium point $\left(E_{2}\right)$ stable, if $r_{1} a_{2}<b_{2} r_{2} k$ and $c_{3} r_{2} k>c_{1} a_{2}$.

iii. Jacobi matrix on equilibrium point $E_{a}=\left(\frac{r_{1}}{b_{1}}, 0,0\right)$ is

$$
l=\left[\begin{array}{ccc}
-r_{1} & -\frac{r_{1} b_{2}}{b_{1}} & -\frac{r_{1} b_{2}}{b_{1}} \\
0 & r_{2} & 0 \\
0 & 0 & -c_{1}+\frac{c_{2} r_{1}}{b_{1}}
\end{array}\right] \text {. }
$$

has eigen values

$\lambda_{1}=-r_{1}, \lambda_{2}=r_{2}, \lambda_{a}=-c_{1}+\frac{c_{3} r_{1}}{b_{1}}$ so

$E_{a}$ unstable.

iv. Jacobi matrix on equilibrium point $E_{4}=\left(0, \frac{r_{2} k}{\sigma_{2}}, \frac{c_{3} r_{2} k-c_{1} \omega_{2}}{c_{4} \sigma_{2}}\right)$ is

$l=\left[\begin{array}{ccc}r_{1}-b_{2} y_{4}-b_{2} z_{4} & 0 & 0 \\ \frac{r_{2}{ }^{2}}{a_{2}} & -r_{2} & 0 \\ c_{2} z_{4} & c_{2} z_{4} & -c_{1}-2 c_{4} z_{4}+c_{2} z_{4}\end{array}\right]$.

because $\quad x=0, r_{2} \frac{\mathbb{2}_{2}}{x+k} y=0$, and

$-c_{1}+c_{2} x+c_{2} y-c_{4} z=0$. so $E_{4}$ sta-

ble if

$\lambda_{1}=r_{1}-\frac{b_{2} k r_{2}}{a_{2}}-\frac{b_{a}}{a_{2} c_{4}}\left(k r_{2} c_{3}-a_{2} c_{1}\right)<0$,

$\frac{r_{1} a_{2} c_{4}-b_{2} k r_{2} c_{4}-b_{2} k r_{2} c_{3}+b_{3} a_{2} c_{1}}{a_{2} c_{4}}<0$.

$c_{4} r_{1}+c_{1} b_{a}<\frac{k r_{2}}{a_{2}}\left(b_{2} c_{4}+b_{3} c_{2}\right)$.
The equilibrium point

$\lambda_{1}=r_{2}>0$ so unstable I.

vi. Jacobi matrix on the equilibrium point $E_{6}=\left(\frac{r_{1} a_{2}-b_{2} r_{2} k}{a_{2} b_{1}+b_{2} r_{2}}, \frac{r_{2}\left(r_{1}+b_{1} k\right)}{a_{2} b_{1}+b_{2} r_{2}}, 0\right)$

$I\left(E_{6}\right)=\left[\begin{array}{ccc}r_{1}-2 b_{1} x_{6}-b_{2} y_{6} & -b_{2} x_{6} & -b_{2} x_{6} \\ \frac{a_{2} y^{2}}{(x+k)^{2}} & r_{2} \frac{2 a_{2} y}{x+k} & 0 \\ 0 & 0 & -c_{1}+c_{2} x_{6}+c_{2} y_{6}\end{array}\right]$.

with,

$r_{1}-b_{1} x-b_{2} y-b_{2} z=0 \Rightarrow r_{1}-b_{1} x_{6}-b_{2} y_{6}=0$

$r_{2} \frac{a_{2}}{x+k} y=0 \Rightarrow \frac{y}{x+k}=\frac{r_{2}}{a_{2}} \rightarrow\left(\frac{y}{x+k}\right)^{2}=\frac{r_{2}^{2}}{a_{2}{ }^{2}}$ $z=0$,

so

$I\left(E_{6}\right)=\left[\begin{array}{ccc}-b_{1} x_{6} & -b_{2} x & -b_{2} x \\ \frac{r_{2}^{2}}{a_{2}} & -\frac{a_{2} y}{x+k} & 0 \\ 0 & 0 & -c_{1}+c_{2} x_{6}+c_{2} y\end{array}\right]$,
$\left|J\left(E_{6}\right)-\lambda I\right|=\left|\begin{array}{ccc}-b_{1} x_{6}-\lambda & -b_{2} x & -b_{3} x \\ \frac{r_{2}^{2}}{a_{2}} & -\frac{a_{2} y}{x+k}-\lambda & 0 \\ 0 & 0 & J_{39}-\lambda\end{array}\right|$,

$=\left(J_{3 a}-\lambda\right)\left[\left(\lambda+b_{1} x\right)\left(\lambda+\frac{a_{2} y}{x+k}\right)+\frac{b_{2} r_{2} J^{2}}{a_{2}} x\right]=0$,

$=\left(J_{3 \mathrm{~B}}-\lambda\right)\left[\lambda^{2}+\left(b_{1} x+\frac{a_{2} y}{x+k}\right) \lambda+\frac{b_{1} a_{2} y}{x+k}+\frac{b_{2} r_{2}^{2}}{a_{2}} x\right]=0$.

result $\lambda_{1}<0$, with used characeristic as follow $\lambda_{1} \lambda_{2}>0 \Lambda \lambda_{1}+\lambda_{2}<0$ proven that $E_{6}$ unstable. v. Jacobi matrix on the equilibrium point

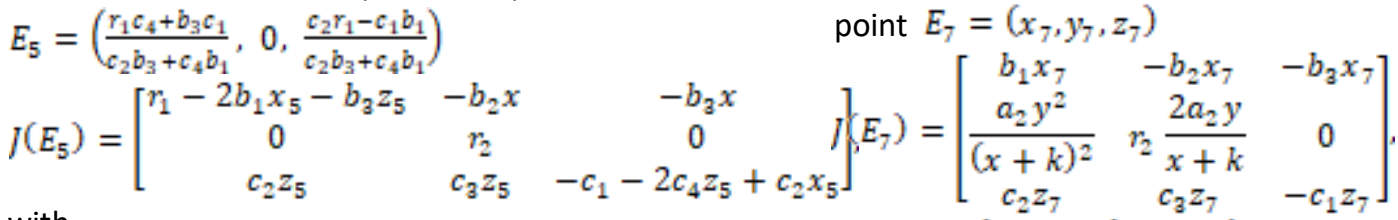

with,
$r_{1}-b_{1} x-b_{2} y-b_{2} z=0 \Rightarrow r_{1}-b_{1} x_{5}-b_{2} z_{5}=0$,

$y=0$,

$-c_{1}+c_{2} x+c_{2} y-c_{4} z=0 \Rightarrow-c_{1}+c_{2} x_{5}-c_{4} z_{5}=0$,

so

$f\left(E_{5}\right)=\left[\begin{array}{ccc}-b_{1} x_{5} & -b_{2} x & -b_{2} x \\ 0 & r_{2} & 0 \\ c_{2} z_{5} & c_{2} z_{5} & -c_{4} z_{5}\end{array}\right]$

$J\left(E_{5}\right)-\lambda I|=| \begin{array}{ccc}-b_{1} x_{5}-\lambda & -b_{2} x & -b_{2} x \\ 0 & x_{2}-\lambda & 0 \\ c_{2} z_{5} & c_{2} z_{5} & -c_{4} z_{5}-\lambda\end{array} \mid$.

$=\left(r_{2}-\lambda_{1}\right)\left|\begin{array}{cc}-b_{1} x_{5}-\lambda & -b_{2} x \\ c_{2} z_{5} & -c_{4} z_{5}-\lambda\end{array}\right|=0$. vii. Jacobi matrix on equilibrium point $E_{7}=\left(x_{7}, y_{7}, z_{7}\right)$

$f\left(E_{7}\right)=\left[\begin{array}{ccc}-b_{1} x_{7} & -b_{2} x & -b_{2} x \\ \frac{r_{2}^{2}}{a_{2}} & -r_{2} & 0 \\ c_{2} z_{7} & c_{2} z_{7} & -c_{1} z_{7}\end{array}\right]$

Same of characteristic with use cofactor expansion second line,

$\left|J\left(E_{7}\right)-\lambda I\right|=\left|\begin{array}{ccc}-b_{1} x_{7}-\lambda & -b_{2} x_{7} & -b_{3} x_{7} \\ \frac{r_{2}^{2}}{a_{2}} & -r_{2}-\lambda & 0 \\ c_{2} z_{7} & c_{3} z_{7} & -c_{1} z_{7}-\lambda\end{array}\right|$. 
(Exviani et al)

$$
\begin{gathered}
=-\frac{r_{2}^{2}}{a_{2}}\left|\begin{array}{cc}
-b_{2} x_{7} & -b_{3} x_{7} \\
c_{2} z_{7} & -c_{1} z_{7}-\lambda
\end{array}\right|+\left(-r_{2}-\lambda\right)\left|\begin{array}{cc}
-b_{1} x_{7}-\lambda & -b_{3} x_{7} \\
c_{2} z_{7} & -c_{1} z_{7}-\lambda
\end{array}\right|=0, \\
=-\frac{1}{a_{2}}\left(a_{2} b_{1} c_{4} \lambda x_{7} z_{7}+a_{2} b_{1} c_{4} x_{2} x_{7} z_{7}+a_{2} b_{3} c_{2} \lambda x_{7} z_{7}+a_{2} b_{3} c_{2} r_{2} x_{7} z_{7}\right. \\
+b_{2} c_{4} x_{2}^{2} x_{7} z_{7}+b_{3} c_{3} r_{2}^{2} x_{7} z_{7}+a_{2} b_{1} \lambda^{2} x_{7}+a_{2} b_{1} \lambda r_{2} x_{9}+a_{2} c_{4} \lambda^{2} z_{7} \\
\left.+a_{2} c_{4} \lambda r_{2} z_{7}+b_{2} \lambda r_{2}^{2} x_{7}+a_{2} \lambda^{3}+a_{2} \lambda^{2} r_{2}\right)_{x}
\end{gathered}
$$

With declaration as:

$$
\begin{gathered}
\lambda^{3}+A \lambda^{2}+B \lambda+C=0, \\
\text { with, } \\
A=c_{4} z_{7}+b_{1} x_{7}+r_{2}>0, \\
B=b_{1} c_{4} x_{7} z_{7}+b_{3} c_{2} x_{7} z_{7}+b_{1} x_{2} x_{9}+c_{4} x_{2} z_{7}+a_{2} x_{2}{ }^{2} x_{7}>0, \\
a_{2}
\end{gathered}
$$

Based on criteria Routh-Hurwitz, the equilibrium point $E_{7}$ local asymptotically stable if $A>0, C>0$ and $A B-C>0$.

\section{Numerical Simulation}

Numerical simulations are performed to see the validity of numerical analysis using the fourth-orde Runge-Kutta method to ilustrate the results of the analysis. There are several cases that are simulated in the discussion of this study, as follows.

\section{Simulation I}

Simulation I (Fig. 1) show $E_{2}$ exists, and conditions of stable $E_{2}$ are $r_{1} a_{2}<b_{2} k r_{2}$ and $c_{1} a_{2}>c_{3} k r_{2}$, Parameter being used $r_{1}=0.5, b_{1}=0.05, b_{2}=0.6, b_{3}=0.1, r_{2}=$ $0.7, a_{2}=0.2, k=0.3, c_{1}=0.9, c_{2}=0.25$, $c_{3}=0.2, c_{4}=0.2$

Thus, the equilibrium points of $E_{1}$ exists, $E_{2}$ exist, $E_{3}$ exists, and $E_{5}$ exists. The numerical simulation to equilibrium point $E_{2}=(0,1,05,0)$. This is relevant to the analysis result which states that equilibrium $E_{2}$ is stable.

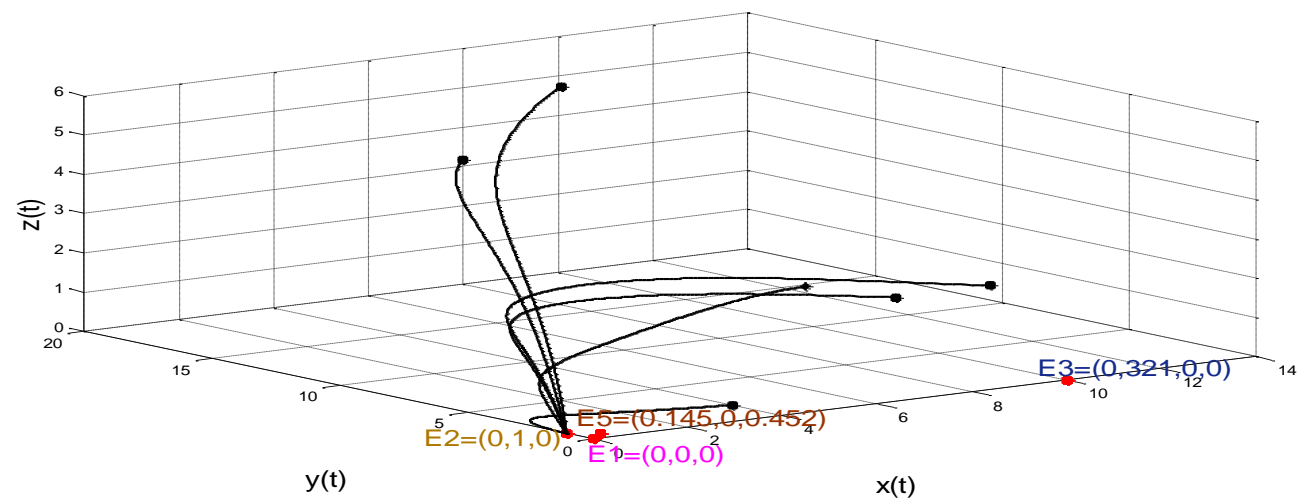

Figure 1. Portrait Phase System Equation 3.1 for simulation I

\section{Simulation II}

Simulation II, the stability conditions of $E_{2}$ are changed into $r_{1} a_{2}>b_{2} k r_{2}$ and $c_{1} a_{2}<c_{3} k r_{2}$, parameter

$r_{1}=0.8, b_{2}=0.4, b_{3}=0.1, b_{1}=0.05, r_{2}=$ $0.7, a_{2}=0.2, k=0.3, c_{1}=0.3, c_{2}=0.25$, $c_{3}=0.6, c_{4}=0.2$. Thus, it produces $E_{4}$ exists, $E_{5}$ exists, $E_{6}$ exists. Then, $E_{7}$ exists and is stable toward equilibrium point, so the initial value shows that $E_{p}$ is stable. 


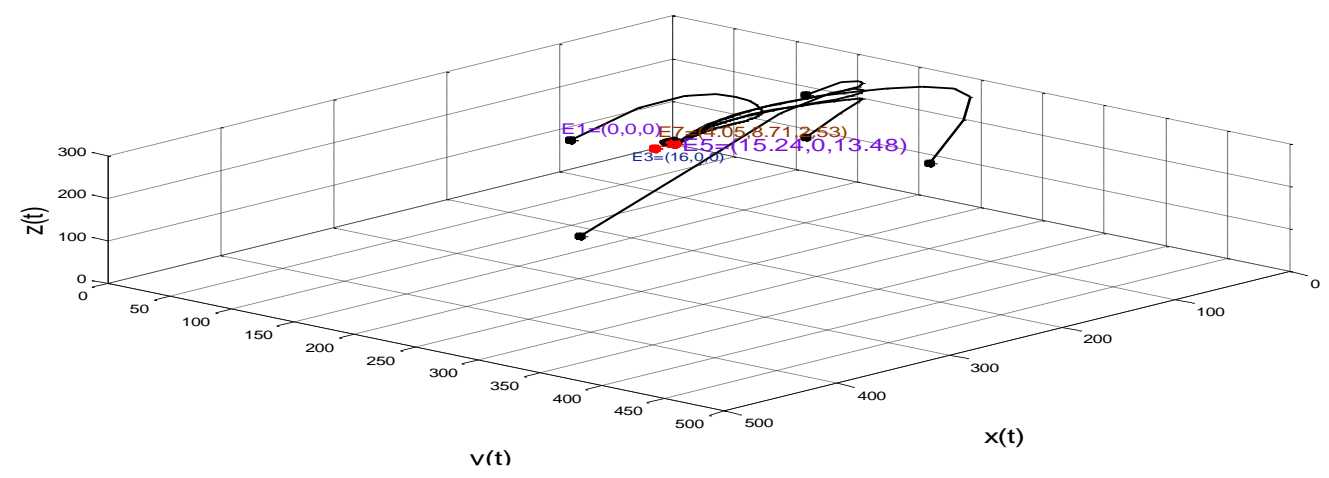

Figure 2. Portrait Phase System Equation 3.1 for Simulation II

\section{Simulation III}

The stability conditions in simulation III of $E_{2}$ are changed into $r_{1} a_{2}>b_{2} k r_{2}$ dan $c_{1} a_{2}>c_{3} k r_{2}$, parameter $r_{1}=0.8, b_{2}=0.4, b_{3}=0.1, b_{1}=0.05, r_{2}=$ $0.7, a_{2}=0.2, k=0.3, c_{1}=0.9, c_{2}=0.25$, $c_{3}=0.2, c_{4}=0.2$. Thus, it produces $E_{1}$ exists, $E_{2}$ exists, $E_{3}$ exists, $E_{5}$ exists, and $E_{6}$ exists, but, in this case, it does not go to any point, so it exists but is unstable.

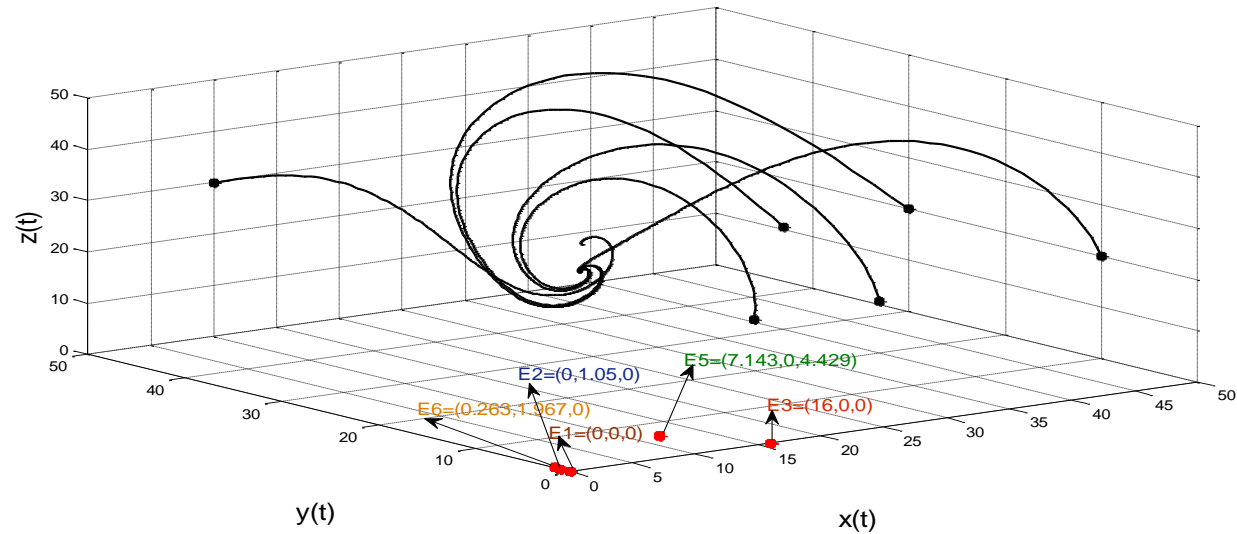

Figure 3. Portrait Phase System Equation 3.1 for Simulation III

\section{Simmulation IV}

The stability conditions in simulation IV, of $E_{2}$ are changed into $r_{1} a_{2}<b_{2} k r_{2}$ dan $c_{1} a_{2}<c_{3} k r_{2}$, parameter $r_{1}=0.5, b_{2}=0.6, b_{3}=0.1, b_{1}=0.05$, $r_{2}=0.7, a_{2}=0.2, k=0.3, c_{1}=0.3, c_{2}=$ 0.25 ,

$c_{3}=0.6, c_{4}=0.2$. Thus, it produces $E_{1}$ exists, $E_{2}$ exists, $E_{3}$ exists, $E_{4}$ exists, and $E_{5}$ exists and unstable.

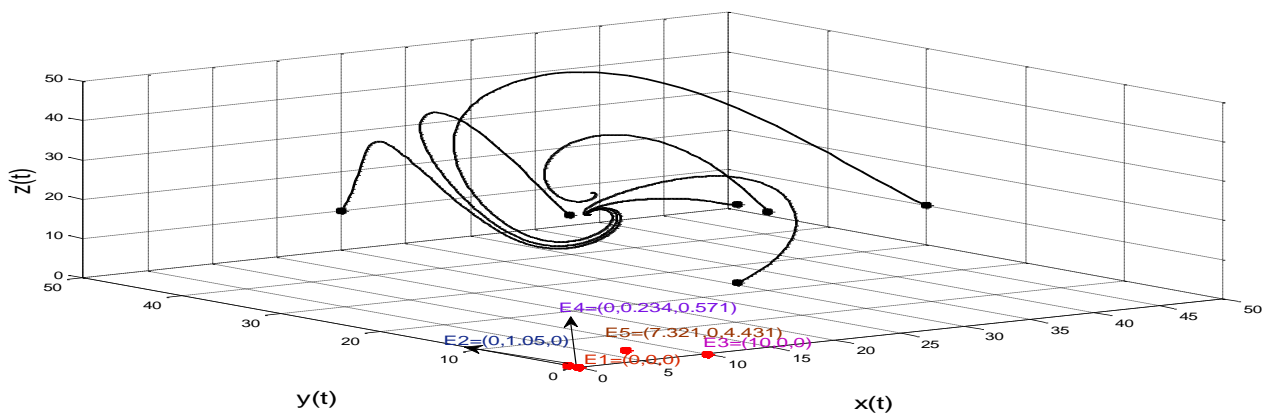

Figure 4. portrait phase system equation 3.1 for simulation IV 
Simulation V:

Stability

condition

$c_{4} r_{1}+c_{1} b_{3}<\frac{r_{2} k}{a_{2}}\left(b_{2} c_{4}+b_{3} c_{3}\right)$, the fifth simulation is conducted to show the stability characteristics of equilibrium point $E_{4}$. Based on the existence condition and the stability of equilibrium point $\quad E_{4^{x}}$ parameter $r_{1}=0.5, b_{2}=0.4, b_{3}=0.1, b_{1}=0.05, r_{2}=$ $0.9, a_{2}=0.2, k=0.5, c_{1}=0.3, c_{2}=0.25$, $c_{3}=0.6, c_{4}=0.2$. Thus, it produces $E_{1}$ exists, $E_{2}$ exists, $E_{3}$ exists, $E_{4}$ exists, and $E_{5}$ exists towards equilibrium point $E_{4 \times}$ This is relevant to the analysis result which states that the equilibrium point is stable.

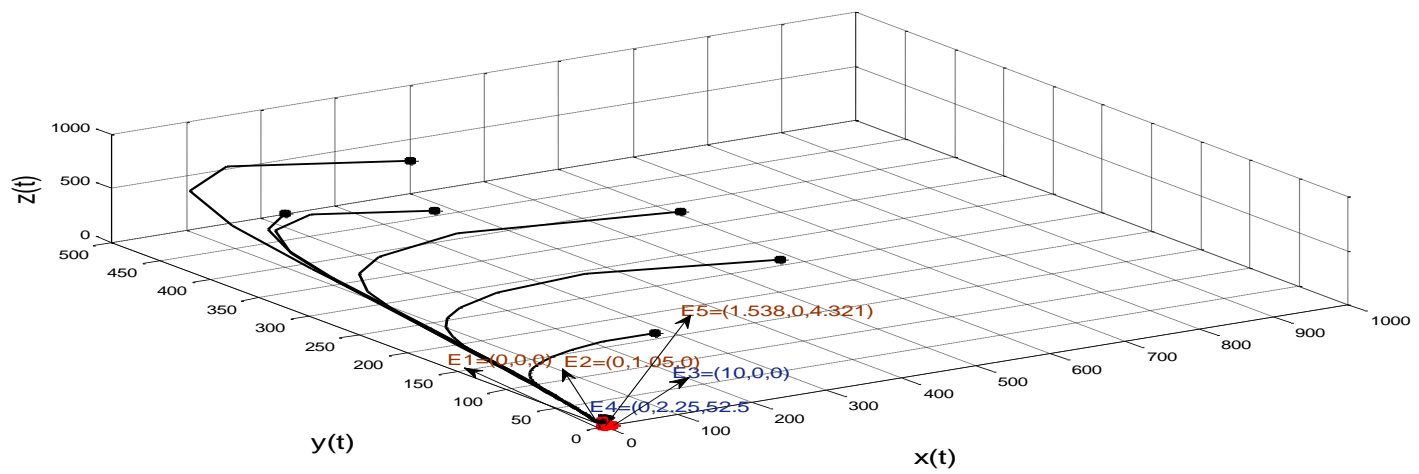

Figure 5. Portrait Phase System Equation 3.1 for Simulation $V$

\section{CONCLUSION}

The conclusions that are drawn based on the discussion of the thesis are as follows. Predatorprey model by Leslie-Gower with omnivore is obtained in a form of common differential equation system. There are seven equilibrium points in the model, there are three of them, i.e. $E_{1}, E_{2}$ and $E_{a}$, unconditionally exist and the other four, i.e. $E_{4}, E_{5}, E_{6}$ and $E_{7}$, conditionally exist. Of the seven equilibrium points, three of them, $E_{1 x}$ $E_{2}, E_{4}$ dan $E_{7}$, have stability condition.

\section{REFERENCES}

[1] Jeffrey, R. C. 2012. Introduction to numerical methods. Lecture Notes for Matth 3311. The Hong Kong University of Science and Technology. Kowloon, Hongkong.

[2] Murray, J.D. 2002. Mathematical biology I: an introduction, $3^{\text {rd }}$ Ed. Springer. New York.

[3] Mohammadi, H., M. Mahzoon. 2013. Effect of weak prey in Leslie-Gower Predator-Prey Model. Appl. Math. Comput. 224, 196-204.

[4] Andayani, P., W.M. Kusumawinahyu. 2015. Global Stability Analysis on a Predator-Prey Model with Omnivora. Appl. Math. Sci. 9(36). 1771-1782.

[5] Previte, J.P., K.A. Hoffman. 2010. Chaos in Predator-Prey Model with an Omnivore. Math. Biol. 92(1). 1-7.

[6] Huo, H.F., X. Wang, C. Carlos. 2011. Dynamics of a stage structured Leslie-
Gower Predator- Prey Model. Appl. Math. Article ID 149341.

[7] Chen, L., F. Chen. 2009. Global stability in Leslie-Gower Predator-prey model With feedback controls. 22(9). 1330-1334.

[8] Tanabe, K., T. Namba. 2005. Omnivory creates chaos in simple food web models. Ecology. 86(12). 3411-3414.

[9] Nagle, R.K., E.B. Saff, A. D. Snider. 2004. Fundamentals of differential equations and boundary value problems, $6^{\text {th }} \mathrm{Ed}$. Pearson Education Inc. USA.

[10] Mattheij, R., J. Molenaar. 2002. Ordinary differential equation in theory and practice. Society for Industrial and Applied Mathematics. New York.

[11] Korobeinikov, A. 2001. A Lyapunov Function for Leslie-Gower Predator-Prey Models. Appl. Math. Lett. 14(6). 697-699.

[12] Polis, G.A., C.A. Myers, R.D. Holt. 1989. The ecology and evolution of intraguild predation: potential competitors that eat each other. Annu. Rev. Ecol. Evol. Syst. 20(1). 297-330. 